
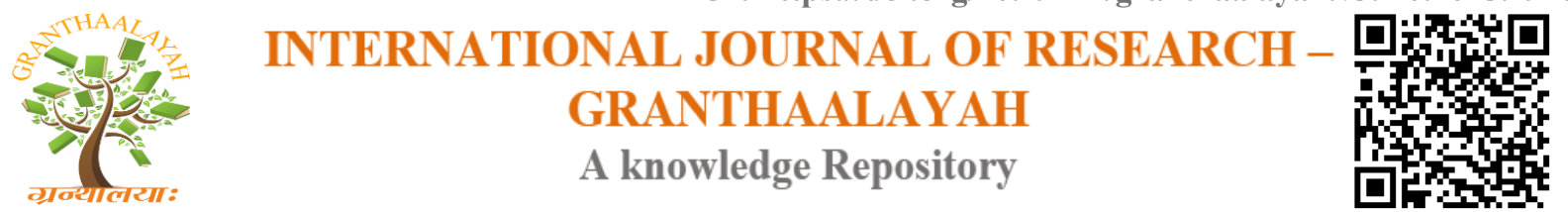

Science

\title{
ON GENERALIGATIONS OF PARTITION FUNCTIONS
}

\author{
Nil Ratan Bhattacharjee *1, Sabuj Das ${ }^{2}$ \\ ${ }^{1}$ Department of Mathematics, University of Chittagong, BANGLADESH \\ ${ }^{2}$ Senior Lecturer, Department of Mathematics Raozan University College, BANGLADESH
}

\begin{abstract}
In 1742, Leonhard Euler invented the generating function for P(n). Godfrey Harold Hardy said Srinivasa Ramanujan was the first, and up to now the only, Mathematician to discover any such properties of $P(n)$. In 1916, Ramanujan defined the generating functions for $X(n), Y(n)$. In 2014, Sabuj developed the generating functions for. In 2005, George E. Andrews found the generating functions for In 1916, Ramanujan showed the generating functions for, , and . This article shows how to prove the Theorems with the help of various auxiliary functions collected from Ramanujan's Lost Notebook. In 1967, George E. Andrews defined the generating functions for P1r (n) and P2r (n). In this article these generating functions are discussed elaborately. This article shows how to prove the theorem $P 2 r(n)=P 3 r(n)$ with a numerical example when $n=9$ and $r=2$. In 1995, Fokkink, Fokkink and Wang defined the in terms of, where is the smallest part of partition. In 2013, Andrews, Garvan and Liang extended the $F F W$-function and defined the generating function for $F F W(z, n)$ in differnt way.
\end{abstract}

Keywords:

FFW-function; Operator; Rogers- Ramanujan Identities;Ramanujan's Lost Notebook.

Cite This Article: Nil Ratan Bhattacharjee, and Sabuj Das, "ON GENERALIGATIONS OF PARTITION FUNCTIONS" International Journal of Research - Granthaalayah, Vol. 3, No. 10(2015): 1-29. DOI: https://doi.org/10.29121/granthaalayah.v3.i10.2015.2928.

\section{INTRODUCTION}

In this article, we give some related definitions of $\mathrm{P}(\mathrm{n}), P^{o}(n), P^{d}(n), \mathrm{X}(\mathrm{n}), \mathrm{Y}(\mathrm{n})$, $X^{1}(n), Y^{1}(n)$ and $P_{\mathrm{e}}^{\mathrm{d}}(n), C^{\prime}(n), C^{\prime \prime}(n), C_{1}^{\prime}(n), C_{1}^{\prime \prime}(n), P^{\prime}(n), P^{\prime \prime}(n), P_{1}^{r}(n), P_{2}^{r}(n), P_{3}^{r}(n), F F W(n)$, $\mathrm{d}(\mathrm{n}),(x)_{\infty},\left(\mathrm{x}^{2} ; \mathrm{x}\right)_{\infty},(z x)_{\infty},(x)_{k}$ and $\left(x^{k+1} ; x\right)_{\infty}$. In section 1.3 , we generate the generating function for $\mathrm{P}(\mathrm{n})$. In section 1.4, we give the generating functions for $P^{o}(n), P^{d}(n)$, and prove the Corollary 1.1 by mathematical expression, and prove the Theorem 1.1 with an example. In section 1.5 , we generate the generating functions for $\mathrm{X}(\mathrm{n}), \mathrm{Y}(\mathrm{n})$ and prove the Corollary 1.2 by mathematical expression and prove the Remark 1.1 with an example. In section 1.6 we develop the generating functions for $X^{1}(n), Y^{1}(n)$ and $\mathrm{P}_{\mathrm{e}}^{\mathrm{d}}(n)$ and prove the Corollary 1.3 in terms of $X^{1}(n), Y^{1}(n)$ and 
prove the another Corollary 1.4 in terms of $\mathrm{P}_{\mathrm{e}}^{\mathrm{d}}(n)$ and $X^{1}(n) \quad$.In section 1.7, we discuss the generating functions for $C^{\prime}(n), C^{\prime \prime}(n)$, and quite some special functions collected from Ramanujan's lost notebook and unpublished papers, and prove the Theorem 1.2 with an example. In section 1.8, we give the generating functions for $C_{1}^{\prime}(n), C_{1}^{\prime \prime}(n)$, and prove the Theorem 1.3 with example. In section 1.9, we give the generating functions for $P^{\prime}(n), P^{\prime \prime}(n)$ and prove the Corollary 1.5 in terms of $P^{\prime}(n), P^{\prime \prime}(n)$ and prove the Theorem 1.4 with an example. In section 1.10 , we generate the generating functions for $P_{1}^{2}(n), P_{2}^{2}(n), P_{2}^{r}(n), P_{3}^{r}(n)$ and prove the Corollary 1.6 in terms of $P_{1}^{r}(n), P_{2}^{r}(n)$, and prove the Theorem 1.5 with an example. Finally in section 1.11 , we discuss the generating function for FFW (n) and give the Relation 1.1 in terms of $F F W(n), \mathrm{d}(\mathrm{n})$, and prove the Corollaries 1.7, 1.8, 1.9 and 1.10 with the help of their generating functions.

\subsection{SOME RELATED DEFINITIONS}

$\mathrm{P}(\mathrm{n})$ : The number of partitions of $\mathrm{n}$. Example: $4,3+1,2+2,2+1+1,1+1+1+1 \therefore \mathrm{P}(4)=5$.

$P^{o}(n)$ : The number of partitions of $n$ into odd parts.

$P^{d}(n)$ :The number of partitions of $n$ into distinct parts.

$\mathrm{X}(\mathrm{n})$ : The number of partitions of $\mathrm{n}$ with no part repeated more than twice.

$\mathrm{Y}(\mathrm{n})$ :The number of partitions of $\mathrm{n}$ with no part divisible by 3 .

$X^{1}(n)$ : The number of partitions of $n$ with no part repeated more than thrice .

$Y^{1}(n)$ : The number of partitions of $n$ with no part divisible by 4 .

$P_{e}^{d}(n)$ : The number of partitions of $n$ into even distinct parts.

$C^{\prime}(n)$ : The number of partitions of $n$ into parts of the forms $5 m+1$ and $5 m+4$.

$C^{\prime \prime}(n)$ : The number of partitions of $n$ into parts of the forms $5 m+2$ and $5 m+3$.

$C_{1}^{\prime}(n)$ : The number of partitions of $n$ into parts without repetitions or parts whose minimal difference is 2 .

$C_{1}^{\prime \prime}(n)$ : The number of partitions of $n$ into parts not less than 2 and with minimal difference 2.

$P^{\prime}(n)$ :The number of partitions of $n$ into parts of the form $n=a_{1}+a_{2}+\ldots+a_{r}$, where $a_{i}-a_{i+1} \geq 3$ and if $3 \mid a_{i}$, then $a_{i}-a_{i+1}>3$

$P^{\prime \prime}(n)$ : The number of partitions of $n$ into parts congruent to $\pm 1(\bmod 6)$.

$P_{1}^{r}(n)$ : The number of partitions of $n$ into part that are either even or not congruent to $4 r-2(\bmod 4 r)$ or odd and congruent to $2 r-1,4 r-1(\bmod 4 r)$.

$P_{2}^{r}(n)$ : The number of partitions of $n$ into parts that are either even or else congruent to $2 r-1(\bmod 2 r)$ with the further restriction that only even parts may be repeated.

$P_{3}{ }^{r}(n)$ : The number of partitions of $n$ of the form $n=b_{1}+b_{2}+\ldots+b_{s}$, where $b_{i} \geq b_{i+1}$, and for $b_{i}$ odd, $b_{i}-b_{i+1} \geq 2 r-1 \quad\left(1 \leq i \leq s\right.$, where $\left.b_{s+1}=0\right)$. 
FFW (n): Let D denote the set of partitions into distinct parts. We define;

$$
\operatorname{FFW}(\mathrm{n})=\sum_{\substack{\pi \in D \\|\pi|=n}}(-1)^{\#(\pi)-1} s(\pi),
$$

where $\mathrm{s}(\pi)$ is the smallest part of a partition $\pi$, and \# $(\pi)$ is the number of parts .

$\mathrm{d}(\mathrm{n})$ : The number of positive divisors of $\mathrm{n}$ like $\mathrm{d}(1)=1, \mathrm{~d}(2)=2, \mathrm{~d}(3)=2, .$.

\section{Product Notations:}

$$
\begin{aligned}
& (x ; x)_{\infty}=(x)_{\infty}=(1-x)\left(1-x^{2}\right)\left(1-x^{3}\right) \ldots \\
& \left(x^{2} ; x\right)_{\infty}=\left(1-x^{2}\right)\left(1-x^{3}\right) \ldots \\
& (z x)_{\infty}=(1-z x)\left(1-z x^{2}\right) \ldots . \\
& (x)_{k}=(1-x)\left(1-x^{2}\right) \ldots\left(1-x^{k}\right) \\
& \left(x^{k+1} ; x\right)_{\infty}=\left(1-x^{k+1}\right)\left(1-x^{k+2}\right) \ldots .
\end{aligned}
$$

\subsection{PARTITION}

A partition of $n$ is a division of $n$ into any number of positive integral parts. Then the sum of the integral parts or summands is $n$. The order of the parts and arrangement in a division of $n$ are irrelevant and the parts are arranged in descending order. Usually a partition of $n$ is denoted by Greek letters $\pi$. We denote the number of partitions of $n$ by $P(n)$. It is convenient to define $P(0)=1$ and,$P(n)=0$ for negative integer of $n$.

Let $A=\left\{a_{1}, a_{2}, \ldots, a_{r}, \ldots\right\}$ be a finite or infinite set of positive integers. If $a_{1}+a_{2}+\ldots+a_{r}=n$, with $a_{r} \in A(r=1,2,3, \ldots)$. Here repetitions are allowed. Then we say that the sum $a_{1}+a_{2}+\ldots+a_{r}$ is a partition of $n$ into parts belonging to the set $A$. So that, $3+2+1$ is a partition of 6 . If a partition contains $p$ numbers, it is called a partition of $n$ into $p$ parts or shortly a $p$-partition of $n$. Hence, $9=$ $4+2+1+1+1$, and we can say that, $4+2+1+1+1$ is a 5 -partition of 9 . The order of the parts is irrelevant, the parts to be arranged in descending order of magnitude, $a_{1} \geq a_{2} \geq a_{3} \geq \ldots a_{r} \geq 1$.

Now explain how to find all the partitions of 7 as follows:

First take 7; then 6 allowed by 1 ; then 5 allowed by all the partitions of 2 (i.e., 2, 1+1); then 4 allowed by all the partitions of 3 (i.e., $3,2+1,1+1+1$ ); then 3 allowed by all the partitions of 4 , which contain no part greater than 3 (i.e., $3+1,2+1+1,1+1+1+1,2+2$ ); then 2 allowed by all the partitions of 5 , which contain no part greater than 2 (i.e., $2+2+1,2+1+1+1,1+1+1+1+1$ ); lastly $1+1+1+1+1+1+1$. Hence the complete set is;

$7, \quad 6+1, \quad 5+2, \quad 5+1+1, \quad 4+3, \quad 4+2+1, \quad 4+1+1+1, \quad 3+3+1, \quad 3+2+1+1, \quad 3+1+1+1+1, \quad 3+2+2$, $2+2+2+1,2+2+1+1+1,2+1+1+1+1+1,1+1+1+1+1+1+1$.

\subsubsection{Generating function for $P(n)$ [Euler (1742)]}

$P(n)$ is the number of partitions of $n$ given in Table-1.1

$$
n \quad \text { Type of partitions }
$$




$\begin{array}{lll}1 & 1 & 1 \\ 2 & 2,1+1 & 2 \\ 3 & 3,2+1,1+1+1 & 3 \\ 4 & 4,3+1,2+2,2+1+1,1+1+1+1 & 5 \\ 5 & 5,4+1,3+2,3+1+1,2+2+1,2+1+1+1,1+1+1+1+1 & 7 \\ \ldots & \ldots & \ldots\end{array}$

It is convenient to define $P(0)=1$, but $P(n)=0$ for $\mathrm{n}<0$.

We can make an expression as;

$$
\begin{aligned}
& P(0)+P(1) x+P(2) x^{2}+P(3) x^{3}+P(4) x^{4}+P(5) x^{5}+\ldots \\
& =1+1 \cdot x+2 \cdot x^{2}+3 \cdot x^{3}+5 \cdot x^{4}+7 \cdot x^{5}+\ldots \\
& =\left(1+x+x^{2}+\ldots\right)\left(1+x^{2}+x^{4}+\ldots\right)\left(1+x^{3}+x^{6}+\ldots\right) \ldots \\
& =\frac{1}{(1-x)\left(1-x^{2}\right)\left(1-x^{3}\right) \ldots} \\
& =\prod_{i=1}^{\infty} \frac{1}{1-x^{i}} \\
& \therefore \prod_{i=1}^{\infty} \frac{1}{\left(1-x^{i}\right)}=\sum_{n=0}^{\infty} P(n) x^{n} .
\end{aligned}
$$

1.4. Generating Functions for $P^{o}(n)$ and $P^{d}(n)$ :

\section{[Collected from Ramanujan's lost notebook]}

\subsubsection{The generating function for $P^{o}(n)$;}

$P^{o}(n)$ is the number of partitions of $n$ into odd parts given in Table-1.2

$\begin{array}{lll}n & \text { Type of partitions } & P^{o}(n) \\ 1 & 1 & 1 \\ 2 & 1+1 & 1 \\ 3 & 3,1+1+1 & 2 \\ 4 & 3+1,1+1+1+1 & 2 \\ 5 & 5,3+1+1,1+1+1+1+1 & 3 \\ \ldots & \cdots \cdots & \ldots\end{array}$

It is convenient to define $P^{o}(0)=1$.

We can write an expression for $P^{o}(n)$ as;

$$
\begin{aligned}
& P^{o}(0)+P^{o}(1) x+P^{o}(2) x^{2}+P^{o}(3) x^{3}+P^{o}(4) x^{4}+\ldots \\
& =1+1 . x+1 \cdot x^{2}+2 \cdot x^{3}+2 \cdot x^{4}+\ldots \\
& =\frac{1}{(1-x)\left(1-x^{3}\right)\left(1-x^{5}\right) \ldots} \quad \text { [Toh, (2011)] } \\
& =\prod_{i=1}^{\infty} \frac{1}{\left(1-x^{2 i-1}\right)}
\end{aligned}
$$




$$
\therefore \prod_{i=1}^{\infty} \frac{1}{\left(1-x^{2 i-1}\right)}=\sum_{n=0}^{\infty} P^{o}(n) x^{n}
$$

\subsubsection{The generating function for $P^{d}(n)$;}

$P^{d}(n)$ is the number of partitions of $n$ into distinct parts given in Table-1.3

$\begin{array}{lll}n & \text { Type of partitions } & P^{d}(n) \\ 1 & 1 & 1 \\ 2 & 2 & 1 \\ 3 & 3,2+1 & 2 \\ 4 & 4,3+1 & 2 \\ 5 & 5,4+1,3+2 & 3 \\ \ldots & \cdots & \ldots \\ \text { It is convenient to define } P^{d}(0)=1 .\end{array}$

We can write an expression for $P^{d}(n)$ as;

$$
\begin{aligned}
& P^{d}(0)+P^{d}(1) x+P^{d}(2) x^{2}+P^{d}(3) x^{3}+P^{d}(4) x^{4}+\ldots \\
& =1+1 . x+1 . x^{2}+2 \cdot x^{3}+2 \cdot x^{4}+\ldots \\
& =(1+x)\left(1+x^{2}\right)\left(1+x^{3}\right) \ldots \\
& =\prod_{n=1}^{\infty}\left(1+x^{n}\right) \\
\therefore & \prod_{n=1}^{\infty}\left(1+x^{n}\right)=\sum_{n=0}^{\infty} P^{d}(n) x^{n} .
\end{aligned}
$$

Corollary 1.1: $\quad P^{o}(n)=P^{d}(n)$

Proof: From above we get;

$$
\begin{aligned}
\sum_{n=0}^{\infty} P^{o}(n) x^{n} & =\prod_{i=1}^{\infty} \frac{1}{\left(1-x^{2 i-1}\right)} \\
& =\frac{1}{(1-x)\left(1-x^{3}\right)\left(1-x^{5}\right) \ldots} \\
& =\frac{\left(1-x^{2}\right)\left(1-x^{4}\right)\left(1-x^{6}\right)}{(1-x)\left(1-x^{2}\right)\left(1-x^{3}\right)} \ldots \\
& =(1+x)\left(1+x^{2}\right)\left(1+x^{3}\right) \ldots \\
& =\sum_{n=0}^{\infty} P^{d}(n) x^{n} \cdot[\text { by above }]
\end{aligned}
$$

Now equating the coefficient of $x^{n}$ from both sides we get;

$$
P^{o}(n)=P^{d}(n) \text {. Hence The Theorem. }
$$

Theorem 1.1 [Das (2013)]: The number of partitions of $n$ into odd parts is equal to the number of partitions of $n$ into unequal parts. i.e., $P^{o}(n)=P^{d}(n)$. 
Proof: We develop an one to one correspondence between the partitions enumerated by $P^{o}(n)$ and those enumerated by $P^{d}(n)$. We start with any partition of $n$ into odd parts say $n=a_{1}+a_{2}+\ldots+a_{r}$. Among these $r$ odd integers, suppose there are $m$ distinct ones, say $c_{1}, c_{2}, \ldots, c_{m}$ by rearranging notation if necessary. Collecting like terms in the partition of $n$, we get $n=e_{1} c_{1}+e_{2} c_{2}+\ldots+e_{m} c_{m}$. We write each co-efficient $e_{i}$ as a unique sum of distinct powers of 2, and write each $e_{i} c_{i}$ as a sum of terms of the type $2^{t} c_{i}$. This gives $n$ as a partition into distinct parts. Thus we have the one to one correspondence. Such as a number $k$ can be expressed uniquely in the binary scale i.e., as $k=2^{a}+2^{b}+2^{c}+\ldots(0 \leq a<b<c \ldots)$. Hence a partition of $n$ into odd parts can be written as; $n=k_{1} \cdot 1+k_{2} \cdot 3+k_{3} \cdot 5+\ldots$ $=\left(2^{a_{1}}+2^{b_{1}}+\ldots\right) \cdot 1+\left(2^{a_{2}}+2^{b_{2}}+\ldots\right) \cdot 3+\left(2^{a_{3}}+2^{b_{3}}+\ldots\right) \cdot 5+\ldots$

and there is an one to one correspondence between this partitions and the into distinct parts $2^{a_{1}} .1$, $2^{b_{1}} .1, \ldots, 2^{a_{2}} .3,2^{b_{2}} .3, \ldots, 2^{a_{3}} .5,2^{b_{3}} .5, \ldots$

Conversely let $n=a_{1}+a_{2}+\ldots+a_{r}$ be a partition of $n$ into distinct parts. We convert this partition into a partition of $n$ with odd parts. For any even positive integer $m$ can be expressed $2^{j} f(m)$ as a multiple odd integer. As for example; $4=1.2^{2}, 6=3.2^{1}, 10=4+6=1.2^{2}+3.2^{1}$, where $2^{j}$ is the highest power of 2 and $f(m)$ is an odd integer. Suppose there are distinct odd integers among $f\left(a_{1}\right)$ , $f\left(a_{2}\right), \ldots, f\left(a_{r}\right)$. Rearrange the subscripts of necessary so that $f\left(a_{1}\right), f\left(a_{2}\right), \ldots, f\left(a_{s}\right)$ are distinct odd integers, and $f\left(a_{s+1}\right), f\left(a_{s+2}\right), \ldots, f\left(a_{r}\right)$ are duplicates of these. Collecting terms, we can write $n=\sum_{i=1}^{s} c_{i} f\left(a_{i}\right)$ with positive integers coefficients $c_{i}$. The final step is to write each $c_{i} f\left(a_{i}\right)$ in the form $f\left(a_{1}\right)+f\left(a_{2}\right)+\ldots+f\left(a_{i}\right)$ with $c_{i}$ terms in the sum. Thus $n$ is expressed as a sum of odd integers. Clearly our correspondence is onto so that, $P^{o}(n)=P^{d}(n)$.

\section{Numerical Example 1.1:}

We take $n=11$, the list of partitions of 11 into odd parts is given below;

$11=9+1+1=7+3+1=7+1+1+1+1=5+5+1$

$=5+3+1+1+1=5+1+1+1+1+1+1=5+3+3=3+3+3+1+1$

$=3+3+1+1+1+1+1=3+1+1+1+1+1+1+1+1$

$=1+1+1+1+1+1+1+1+1+1+1$.

So there are 12 partitions. i.e., $P^{o}(11)=12$.

Again the list of partitions of 11 into unequal parts is given below;

$11=10+1=9+2=8+3=8+2+1=7+4=7+3+1$

$=6+5=6+4+1=6+3+2=5+4+2=5+3+2+1$.

So there are 12 partitions. i.e., $P^{d}(11)=12$.

Therefore, $P^{o}(11)=P^{d}(11)$.

\subsection{Generating functions for $X(n)$ and $Y(n)$ :}

\subsubsection{The generating function for $X(n)$ :}

\section{[Collected from Ramanujan's lost notebook]}

$X(n)$ is the number of partitions of $n$ with no part repeated more than twice given inTable-1.4 


$\begin{array}{lll}n & \text { Partitions of } n \text { with no part repeated more than twice } & X(n) \\ 1 & 1 & 1 \\ 2 & 2,1+1 & 2 \\ 3 & 3,2+1 & 2 \\ 4 & 4,3+1,2+2,2+1+1 & 4 \\ 5 & 5,4+1,3+2,3+1+1,2+2+1 & 5\end{array}$

We can write an expression for $X(n)$ as;

It is convenient to define $X(0)=1$.

$$
\begin{aligned}
& X(0)+X(1) x+X(2) x^{2}+X(3) x^{3}+X(4) x^{4}+\ldots \\
&=1+1 \cdot x+2 \cdot x^{2}+2 \cdot x^{3}+4 \cdot x^{4}+5 \cdot x^{5}+\ldots \\
&=\left(1+x+x^{2}\right)\left(1+x^{2}+x^{4}\right)\left(1+x^{3}+x^{6}\right) \ldots \\
&=\prod_{n=1}^{\infty}\left(1+x^{n}+x^{2 n}\right) . \\
& \therefore \prod_{n=1}^{\infty}\left(1+x^{n}+x^{2 n}\right)=\sum_{n=0}^{\infty} X(n) x^{n} .
\end{aligned}
$$

1.5.2 Generating function for $Y(n)$ :

$Y(n)$ is the number of partitions of $n$ with no part divisible by 3 given

inTable-1.5

$\begin{array}{lll}n & \text { Partitions of } n \text { with no part divisible by } 3 & Y(n) \\ 1 & 1 & 1 \\ 2 & 2,1+1 & 2 \\ 3 & 2+1,1+1+1 & 2 \\ 4 & 4,2+2,2+1+1,1+1+1+1 & 4 \\ 5 & 5,4+1,2+2+1,2+1+1+1,1+1+1+1+1 & 5 \\ \ldots & \ldots & \ldots\end{array}$

It is convenient to define $Y(0)=1$.

We can write an expression for $Y(n)$ as;

$$
\begin{aligned}
& Y(0)+Y(1) x+Y(2) x^{2}+Y(3) x^{3}+Y(4) x^{4}+Y(5) x^{5}+\ldots \\
& =1+1 . x+2 \cdot x^{2}+2 \cdot x^{3}+4 \cdot x^{4}+5 . x^{5}+\ldots \\
& =\frac{1}{(1-x)\left(1-x^{2}\right)\left(1-x^{4}\right)\left(1-x^{5}\right) \ldots\left(1-x^{3 n-2}\right)\left(1-x^{3 n-1}\right) \ldots \text { [Andrews (1979] }} \\
& =\prod_{n=1}^{\infty} \frac{1}{\left(1-x^{3 n-2}\right)\left(1-x^{3 n-1}\right)} \\
\therefore & \prod_{n=1}^{\infty} \frac{1}{\left(1-x^{3 n-2}\right)\left(1-x^{3 n-1}\right)}=\sum_{n=0}^{\infty} Y(n) x^{n} .
\end{aligned}
$$

Corollary 1.2: $\quad X(n)=Y(n)$

Proof: From above we get; 


$$
\begin{aligned}
& \sum_{n=0}^{\infty} X(n) x^{n}=\prod_{n=1}^{\infty}\left(1+x^{n}+x^{2 n}\right) \\
&=\left(1+x+x^{2}\right)\left(1+x^{2}+x^{4}\right)\left(1+x^{3}+x^{6}\right) \ldots\left(1+x^{n}+x^{2 n}\right) \ldots \infty \\
&=\frac{1-x^{3}}{1-x} \cdot \frac{1-x^{6}}{1-x^{2}} \cdot \frac{1-x^{9}}{1-x^{3}} \ldots \frac{1-x^{3 n}}{1-x^{n}} \ldots \\
&=\frac{1}{(1-x)\left(1-x^{2}\right)\left(1-x^{4}\right)\left(1-x^{5}\right) \ldots\left(1-x^{3 n-2}\right)\left(1-x^{3 n-1}\right) \ldots} \\
&=\prod_{n=1}^{\infty} \frac{1}{\left(1-x^{3 n-2}\right)\left(1-x^{3 n-1}\right)} \\
& \therefore \sum_{n=0}^{\infty} X(n) x^{n}=\sum_{n=0}^{\infty} Y(n) x^{n} .
\end{aligned}
$$

Equating the coefficient of $x^{n}$ from both sides we get;

$$
X(n)=Y(n) \text {. Hence the Corollary. }
$$

Remark 1.1 [Das (2014)]: The number of partitions of $n$ with no part repeated more than twice is equal to the number of partitions of $n$ with no part divisible by 3 .

$$
\text { i.e., } X(n)=Y(n) \text {. }
$$

Proof: We develop a one-to-one correspondence between the partitions enumerated by $X(n)$ and those enumerated by $Y(n)$. Let $n=a_{1}+a_{2}+\ldots+a_{r}$ be a partition of $n$ with no part is repeated more than twice. We transfer this into a partition of $n$ with no part is divisible by 3 . If a part $a_{m}$ of the partition, which is divisible by 3 , enumerated by $X(n)$ can be expressed into three equal parts, such that: $6=2+2+2,3=1+1+1$. Rearranging the parts of the partition, we can say that the parts are not divisible by 3 . Clearly, our correspondence is one-to-one.

Conversely, we start any partition of $n$ into with no part is divisible by 3 , say $n=a_{1}+a_{2}+\ldots+a_{r}$, we consider the same part not less than thrice, it would be unique sum by same three parts by taking a group, such that, $5+1+1+1=5+3$ and $2+2+2+1+1=6+1+1$.

This gives $n$ as a partition with no part is repeated more than twice. Thus, we have the one-to-one correspondence. The corresponding is onto, so that $X(n)=Y(n)$. Hence the Remark.

\section{Numerical Example1.2:}

When $n=8$, the listed partitions of 8 with no part repeated more than twice is given below; $8=7+1=6+2=6+1+1=5+3=5+2+1=4+4=4+3+1=4+2+1+1=4+2+2=3+3+2=3+3+1+1=$ $3+2+2+1$.

So, there are 13 partitions i.e., $X(8)=13$. Again, the list of partitions of 8 with no part is divisible by 3 is given below:

$8=7+1=5+2+1=5+1+1+1+1=4+4=4+2+1+1=4+2+2=4+1+1+1+1=2+2+2+2$ $=2+2+2+1+1=2+2+1+1+1+1=2+1+1+1+1+1+1=1+1+1+1+1+1+1+1$.

So, there are 13 partitions i.e., $Y(8)=13$.

$$
\therefore X(n)=Y(n) \text {. }
$$

1.6. Generating functions for $X^{1}(n), Y^{1}(n)$ and $\mathrm{P}_{\mathrm{e}}^{\mathrm{d}}(n)$ : 


\subsubsection{The generating function for $X^{1}(n)$ :}

$X^{1}(n)$ is the number of partitions of $n$ with no part repeated more than thrice given in Table-1.6

$\begin{array}{lll}n & \text { Partitions of } n \text { with no part repeated more than thrice } & X^{1}(n) \\ 1 & 1 & 1 \\ 2 & 2,1+1 & 2 \\ 3 & 3,2+1,1+1+1 & 3 \\ 4 & 4,3+1,2+2,2+1+1 & 4 \\ 5 & 5,4+1,3+2,3+1+1,2+2+1,2+1+1+1 & 6 \\ \cdots & \cdots & \cdots\end{array}$

We can write an expression for $X^{1}(n)$ as;

$$
\begin{aligned}
& X^{1}(0)+X^{1}(1) x+X^{1}(2) x^{2}+X^{1}(3) x^{3}+X^{1}(4) x^{4}+\ldots \\
= & 1+1 \cdot x+2 \cdot x^{2}+3 x^{3}+4 \cdot x^{4}+6 x^{5}+9 x^{6}+. . \\
& =\left(1+x+x^{2}+x^{3}\right)\left(1+x^{2}+x^{4}+x^{6}\right)\left(1+x^{3}+x^{6}+x^{9}\right) \ldots \\
& =\prod_{n=1}^{\infty}\left(1+x^{n}+x^{2 n}+x^{3 n}\right) . \\
\therefore & \prod_{n=1}^{\infty}\left(1+x^{n}+x^{2 n}+x^{3 n}\right)=\sum_{n=0}^{\infty} X^{1}(n) x^{n} .
\end{aligned}
$$

\subsubsection{Generating function $Y^{1}(n)$ for:}

$Y^{1}(n)$ is the number of partitions of $n$ with no part divisible by 4 given inTable-1.7

$\begin{array}{llc}n & \text { Partitions of } n \text { with no part divisible by } 4 & Y^{1}(n) \\ 1 & 1 & 1 \\ 2 & 2,1+1 & 2 \\ 3 & 3,2+1,1+1+1 & 3 \\ 4 & 3+1,2+2,2+1+1,1+1+1+1 & 4 \\ 5 & 5,3+2,3+1+1,2+2+1,2+1+1+1,1+1+1+1+1 & 6 \\ \cdots & \cdots & \ldots \\ & & \end{array}$

We can write an expression for $Y^{1}(n)$ as;

$$
\begin{aligned}
& Y^{1}(0)+Y^{1}(1) x+Y^{1}(2) x^{2}+Y^{1}(3) x^{3}+Y^{1}(4) x^{4}+Y^{1}(5) x^{5}+\ldots \\
& =1+1 \cdot x+2 \cdot x^{2}+3 \cdot x^{3}+4 \cdot x^{4}+6 \cdot x^{5}+9 x^{5}+\ldots \\
& =\frac{1}{(1-x)\left(1-x^{2}\right)\left(1-x^{3}\right)\left(1-x^{5}\right)\left(1-x^{6}\right)\left(1-x^{7}\right)\left(1-x^{9}\right) \ldots}
\end{aligned}
$$




$$
\begin{aligned}
& =\prod_{n \neq 0(\bmod 4)} \frac{1}{\left(1-x^{n}\right)} \\
\therefore & \prod_{n \neq 0(\bmod 4)} \frac{1}{\left(1-x^{n}\right)}=\sum_{n=0}^{\infty} Y^{1}(n) x^{n} .
\end{aligned}
$$

Corollary 1.3: $\quad X^{1}(n)=Y^{1}(n)$

Proof: From above we get;

$$
\begin{aligned}
& \sum_{n=0}^{\infty} X^{1}(n) x^{n}=\prod_{n=1}^{\infty}\left(1+x^{n}+x^{2 n}+x^{3 n}\right) \\
&=\left(1+x+x^{2}+x^{3}\right)\left(1+x^{2}+x^{4}+x^{6}\right)\left(1+x^{3}+x^{6}+x^{9}\right) \ldots \\
&=\frac{\left(1+x+x^{2}+x^{3}\right)(1-\mathrm{x})}{(1-\mathrm{X})} \cdot \frac{\left(1+x^{2}+x^{4}+x^{6}\right)\left(1-\mathrm{x}^{2}\right)}{\left(1-\mathrm{x}^{2}\right)} \cdot \frac{\left(1+x^{3}+x^{6}+x^{9}\right)\left(1-\mathrm{x}^{3}\right)}{\left(1-\mathrm{x}^{3}\right)} \ldots \\
&=\frac{1-x^{4}}{1-x} \cdot \frac{1-x^{8}}{1-x^{2}} \cdot \frac{1-x^{12}}{1-x^{3}} \ldots \\
&=\frac{1}{(1-x)\left(1-x^{2}\right)\left(1-x^{3}\right)\left(1-x^{5}\right)\left(1-x^{6}\right)\left(1-x^{7}\right)\left(1-x^{9}\right) \ldots} \\
&=\prod_{n \neq 0(\bmod 4)}\left(1-x^{n}\right)=\sum_{n=0}^{\infty} Y^{1}(n) x^{n} . \\
& \therefore \sum_{n=0}^{\infty} X^{1}(n) x^{n}=\sum_{n=0}^{\infty} Y^{1}(n) x^{n} .
\end{aligned}
$$

Equating the coefficient of $x^{n}$ from both sides we get;

$X^{1}(n)=Y^{1}(n)$. Hence the Corollary.

Remark 1.2 [Das (2014)]: The number of partitions of $n$ with no part repeated more than thrice is equal to the number of partitions of $n$ with no part divisible by 4 .

$$
\text { i.e., } X^{1}(n)=Y^{1}(n) \text {. }
$$

Proof: We can prove the Remark very easily as same as the Remark 1.1 by changing four equal terms into a single term.

\subsubsection{The generating function for $P_{e}^{d}(n)$ :}

$P_{e}^{d}(n)$ is the number of partitions of $n$ into even distinct parts given in Table-1.8

$$
\begin{array}{lll}
n & \text { Partitions of } n \text { into even distinct parts } & P_{e}^{d}(n) \\
1 & 1 & 1 \\
2 & 2,1+1 & 2 \\
3 & 3,2+1,1+1+1 & 3 \\
4 & 4,3+1,2+1+1,1+1+1+1 & 4 \\
5 & 5,4+1,3+2,3+1+1,2+1+1+1,1+1+1+1+1 & 6 \\
\cdots & \cdots & \cdots
\end{array}
$$

We can write an expression for $P_{e}^{d}(n)$ as; 


$$
\begin{aligned}
& P_{e}^{d}(0)+P_{e}^{d}(1) x+P_{e}^{d}(2) x^{2}+P_{e}^{d}(3) x^{3}+P_{e}^{d}(4) x^{4}+\ldots \\
&= 1+1 \cdot x+2 \cdot x^{2}+3 \cdot x^{3}+4 \cdot x^{4}+6 x^{5}+9 \cdot x^{6}+\ldots \\
&=\frac{\left(1+x^{2}\right)\left(1+x^{4}\right)\left(1+x^{6}\right)}{(1-\mathrm{x})\left(1-x^{3}\right)\left(1-x^{5}\right)} \ldots \\
&=\prod_{n=1}^{\infty} \frac{\left(1+x^{2 n}\right)}{\left(1-x^{2 n-1}\right)} \\
& \therefore \prod_{n=1}^{\infty} \frac{\left(1+x^{2 n}\right)}{\left(1-x^{2 n-1}\right)}=\sum_{n=0}^{\infty} P_{e}^{d}(n) x^{n} .
\end{aligned}
$$

Corollary 2.4: $\quad P_{e}^{d}(n)=X^{1}(n)$

Proof: From above we get;

$$
\begin{aligned}
& \sum_{n=0}^{\infty} P_{e}^{d}(n) x^{n}=\prod_{n=1}^{\infty} \frac{\left(1+x^{2 n}\right)}{\left(1-x^{2 n-1}\right)} \\
& =\frac{\left(1+x^{2}\right)\left(1+x^{4}\right)\left(1+x^{6}\right)}{(1-\mathrm{x})\left(1-x^{3}\right)\left(1-x^{5}\right)} \cdots \\
& =\frac{\left(1+x^{2}\right)\left(1+x^{4}\right)\left(1+x^{6}\right) .}{(1-\mathrm{x})\left(1-x^{3}\right)\left(1-x^{5}\right) . .} \cdot \frac{\left(1-x^{2}\right)\left(1-x^{4}\right)\left(1-x^{6}\right) .}{\left(1-x^{2}\right)\left(1-x^{4}\right)\left(1-x^{6}\right) .} \text {. } \\
& =\frac{\left(1+x^{2}\right)\left(1-x^{2}\right)\left(1+x^{4}\right)\left(1-x^{4}\right)\left(1+x^{6}\right)\left(1-x^{6}\right) .}{(1-\mathrm{x})\left(1-x^{2}\right)\left(1-x^{3}\right) .} \\
& =\frac{\left(1-x^{4}\right)\left(1-x^{8}\right)\left(1-x^{12}\right) . .}{(1-\mathrm{x})\left(1-x^{2}\right)\left(1-x^{3}\right) . .} \\
& =\left(1+x+x^{2}+x^{3}\right)\left(1+x^{2}+x^{4}+x^{6}\right)\left(1+x^{3}+x^{6}+x^{9}\right) \ldots \\
& =\prod_{n=1}^{\infty}\left(1+x^{n}+x^{2 n}+x^{3 n}\right) \\
& =\sum_{n=0}^{\infty} X^{1}(n) x^{n} \\
& \therefore \sum_{n=0}^{\infty} P_{e}^{d}(n) x^{n}=\sum_{n=0}^{\infty} X^{1}(n) x^{n} \text {. }
\end{aligned}
$$

Now equating the coefficient of $x^{n}$ from both sides we get;

$P_{e}^{d}(n)=X^{1}(n)$. Hence the Corollary.

1.7.Generating functions for $C^{\prime}(n)$ and $C_{1}^{\prime}(n)$ :

[Collected from Ramanujan's lost notebook and Andrews (1979)]

1.7.1 The generating function for $C^{\prime}(n)$ : 
$C^{\prime}(n)$ is the number of partitions of $n$ into parts of the forms $5 m+1$ and $5 m+4$ given inTable-1.9

$n \quad$ Partitions of $n$ into parts of the forms $5 m+1$ and $5 m+4 \quad C^{\prime}(n)$

111

$2 \quad 1+1 \quad 1$

$3 \quad 1+1+1 \quad 1$

$44,1+1+1+1 \quad 2$

$54+1,1+1+1+1+1 \quad 2$

$66,4+1+1,1+1+1+1+1+1 \quad 3$

It is convenient to define $C^{\prime}(0)=1$

We can write an expression for $C^{\prime}(n)$ as;

$$
\begin{aligned}
& C^{\prime}(0)+C^{\prime}(1) x+C^{\prime}(2) x^{2}+C^{\prime}(3) x^{3}+C^{\prime}(4) x^{4}+C^{\prime}(5) x^{5}+\ldots \\
& =1+x+x^{2}+x^{3}+2 x^{4}+2 x^{5}+3 x^{6}+\ldots \\
& =\frac{1}{(1-x)\left(1-x^{4}\right)\left(1-x^{6}\right)\left(1-x^{9}\right) \ldots \infty} \text { [Hardy et al. (1917)] } \\
& =\sum_{m=0}^{\infty} \frac{1}{\left(1-x^{5 m+1}\right)\left(1-x^{5 m+4}\right)} \\
& \therefore \sum_{m=0}^{\infty} \frac{1}{\left(1-x^{5 m+1}\right)\left(1-x^{5 m+4}\right)}=1+\sum_{n=1}^{\infty} C^{\prime}(n) x^{n} .
\end{aligned}
$$

\subsubsection{The generating function for $C_{1}^{\prime}(n)$ :}

$C_{1}^{\prime}(n)$ is the number of partitions of $n$ into parts without repetitions or parts, whose minimal difference is 2 given in Table-1.10

$n \quad$ Partitions of n into parts without repetitions or parts, $\quad C_{1}^{\prime}(n)$

whose minimal difference is 2

11

22

$3 \quad 3$ 
It is convenient to define $C_{1}^{\prime}(0)=1$.

We can write an expression for $C_{1}^{\prime}(n)$ as;

$$
\begin{aligned}
& C_{1}^{\prime}(0)+C_{1}^{\prime}(1) x+C_{1}^{\prime}(2) x^{2}+C_{1}^{\prime}(3) x^{3}+C_{1}^{\prime}(4) x^{4}+C_{1}^{\prime}(5) x^{5}+\ldots \\
& =1+x+x^{2}+x^{3}+2 x^{4}+2 x^{5}+3 x^{6}+3 x^{7}+\ldots \infty \\
& =1+\frac{x}{1-x}+\frac{x^{4}}{(1-x)\left(1-x^{2}\right)}+\frac{x^{9}}{(1-x)\left(1-x^{2}\right)\left(1-x^{3}\right)}+\ldots \infty \quad \text { [Andrews (2005)] } \\
& =1+\sum_{m=1}^{\infty} \frac{x^{m^{2}}}{(1-x)\left(1-x^{2}\right) \ldots\left(1-x^{m}\right)} \\
& \therefore 1+\sum_{m=1}^{\infty} \frac{x^{m^{2}}}{(1-x)\left(1-x^{2}\right) . .\left(1-x^{m}\right)}=\sum_{n=0}^{\infty} C_{1}^{\prime}(n) x^{n} .
\end{aligned}
$$

\subsubsection{In 1916, Ramanujan defined the following series in his Lost Notebook;}

We get; $H_{k}(a, x)=\frac{G_{k}(a, x)}{(1-a)(1-a x) \ldots \infty}$, [where $H_{0}=0$ and $k=1$ or 2$] \ldots$

and

$$
H_{k}=H_{k}(a, x)=\sum_{n=0}^{\infty}(-1)^{n} a^{2 n} x^{\frac{n(5 n+1)-2 k n}{2}}\left(1-a^{k} x^{2 k n}\right) P_{n} Q_{n}(a) \text {, }
$$

where $P_{n}=\prod_{r=1}^{n} \frac{1}{1-x^{r}}$, and $Q_{n}(a)=\prod_{r=n}^{\infty} \frac{1}{1-a x^{r}}$. It is convenient to define $P_{0}=1$.

And $G_{k}(a, x)=\prod_{n=0}^{\infty}(-1)^{n} a^{2 n} x \frac{n(5 n+1)-2 k n}{2}\left(1-a^{k} x^{2 k n}\right) C_{n} \quad$ with $|x|<1$ and $|a|<1$.

where $k$ is 1 or 2 and $C_{0}=1, C_{n}=\frac{(1-a)(1-a x) \ldots\left(1-a x^{n-1}\right)}{(1-x)\left(1-x^{2}\right) \ldots\left(1-x^{n}\right)}$.

$H_{k}-H_{k-1}=a^{k-1} \eta H_{3-k}$, where the operator $\eta$ is defined by $\eta f(a)=f(a x)$, and $k=1$ or 2 .

If $\mathrm{k}=1$ and $\mathrm{a}=\mathrm{x}$ then;

$$
G_{1}(x, x)=1-x-x^{4}+x^{7}+x^{13}-\ldots \infty
$$




$$
=\prod_{n=0}^{\infty}\left(1-x^{5 n+1}\right)\left(1-x^{5 n+4}\right)\left(1-x^{5 n+5}\right)
$$

Again if $\mathrm{k}=2$ and $\mathrm{a}=\mathrm{x}$ then;

$$
\begin{aligned}
G_{2}(x, x) & =1-x^{2}-x^{3}+x^{9}+x^{11}-\ldots \infty \\
& =\prod_{m=0}^{\infty}\left(1-x^{5 m+2}\right)\left(1-x^{5 m+3}\right)\left(1-x^{5 m+5}\right) .
\end{aligned}
$$

Theorem 1.2: The number of partitions of $n$ with minimal difference 2 is equal to the number of partitions of $n$ into parts of the forms $5 m+1$ and $5 m+4$.

$$
\text { i.e., } C_{1}^{\prime}(n)=C^{\prime}(n) \text {. }
$$

Proof: From Ramanujan's Lost Notebook, we get;

$H_{k}(a, x)=\frac{G_{k}(a, x)}{(1-a)(1-a x) \ldots \infty}$, [where $H_{0}=0$ and $k=1$ or 2$]$

If $k=2$ and $a=x$, we get;

$$
\begin{aligned}
H_{2}(x, x)=\frac{G_{2}(x, x)}{(1-x)\left(1-x^{2}\right)\left(1-x^{3}\right) \ldots \infty} & =\frac{\prod_{m=0}^{\infty}\left(1-x^{5 m+2}\right)\left(1-x^{5 m+3}\right)\left(1-x^{5 m+5}\right)}{(1-x)\left(1-x^{2}\right)\left(1-x^{3}\right) \ldots \infty \quad[\mathrm{by}(1.6 .3 \mathrm{~d}]} \\
& =\frac{1}{(1-\mathrm{x})\left(1-x^{4}\right)\left(1-x^{6}\right)\left(1-x^{9}\right)\left(1-x^{11}\right)\left(1-x^{14}\right) \ldots} \\
o r, H_{2}(x, x) & =\prod_{m=0}^{\infty} \frac{1}{\left(1-x^{5 m+1}\right)\left(1-x^{5 m+4}\right)} \quad \ldots \quad
\end{aligned}
$$

Again, from Ramanujan's Lost Notebook, we get;

$H_{k}-H_{k-1}=a^{k-1} \eta H_{3-k}$, where the operator $\eta$ is defined by $\eta f(a)=f(a x)$, and $k=1$ or 2 , then $H_{1}=\eta H_{2}, H_{2}-H_{1}=a \eta H_{1}$.

So we have,

$H_{2}=\eta H_{2}+a \eta^{2} H_{2}$.

We suppose that; $H_{2}=1+c_{1} a+c_{2} a^{2}+\ldots$

where the coefficients $c_{i}$ depend on $x$ only. Substituting this into (1.6.3f), we obtain;

$1+c_{1} a+c_{2} a^{2}+\ldots \infty=1+c_{1} a x+c_{2} a^{2} x^{2}+\ldots \infty+a\left(1+c_{1} a x^{2}+c_{2} a^{2} x^{4}+\ldots \infty\right)$.

Hence, equating the coefficients of various powers of a from both sides we get;

$c_{1}=\frac{1}{1-x}, c_{2}=\frac{x^{2}}{1-x^{2}} c_{1}, c_{3}=\frac{x^{4}}{1-x^{3}} c_{2}, \ldots, c_{n}=\frac{x^{n(n-1)}}{(1-x)\left(1-x^{2}\right) \ldots\left(1-x^{n}\right)}$.

Putting these values in(1.6.3g) 
$H_{2}=H_{2}(a, x)=1+\frac{a}{1-x}+\frac{a^{2} x^{2}}{(1-x)\left(1-x^{2}\right)}+\frac{a^{3} x^{6}}{(1-x)\left(1-x^{2}\right)\left(1-x^{3}\right)}+\ldots \infty \ldots$

If $a=x$, then; $H_{2}(x, x)=1+\frac{x}{1-x}+\frac{x^{4}}{(1-x)\left(1-x^{2}\right)}+\frac{x^{9}}{(1-x)\left(1-x^{2}\right)\left(1-x^{3}\right)}+\ldots$

From (1.6.3e) and (1.6.3i) we get;

$$
\begin{aligned}
& 1+\frac{x}{(1-x)}+\frac{x^{4}}{(1-x)\left(1-x^{2}\right)}+\frac{x^{9}}{(1-x)\left(1-x^{2}\right)\left(1-x^{3}\right)}+\ldots=\prod_{m=0}^{\infty} \frac{1}{\left(1-x^{5 m+1}\right)\left(1-x^{5 m+4}\right)} \\
& \text { i.e, } 1+\sum_{m=1}^{\infty} \frac{x^{m^{2}}}{(1-x)\left(1-x^{2}\right) . .\left(1-x^{m}\right)}=\prod_{m=0}^{\infty} \frac{1}{\left(1-x^{5 m+1}\right)\left(1-x^{5 m+4}\right)}
\end{aligned}
$$

[It is known as Rogers- Ramanujan's Identity]

$$
\therefore 1+\sum_{n=1}^{\infty} C_{1}^{\prime}(n) x^{n}=1+\sum_{n=1}^{\infty} C^{\prime}(n) x^{n} \text {. }
$$

Equating the co-efficient of $\mathrm{x}^{\mathrm{n}}$ from both side we get;

$$
C_{1}^{\prime}(n)=C^{\prime}(n)
$$

i.e., the number of partitions of $n$ with minimal difference 2 is equal to the number of partitions of $n$ into parts of the forms $5 m+1$ and $5 m+4$. Hence the Theorem.

\section{Example 1.3:}

For $n=11$, there are 7 partitions of 11 that are enumerated by $C_{1}^{\prime}(n)$ of above statement, which are given bellow;

$11,10+1,9+2,8+3,7+4,7+3+1,6+4+1, \therefore C_{1}^{\prime}(11)=7$.

There are 7 partitions of 11 are enumerated by $C_{1}^{\prime}(n)$ of above statement, which are given bellow:

$11,9+1+1,6+4+1,6+1+1+1+1+1,4+4+1+1+1,4+1+1+1+1+1+1+1$, $1+1+1+1+1+1+1+1+1+1+1, \quad \therefore \quad C^{\prime}(11)=7$.

Hence, $C_{1}^{\prime}(11)=C^{\prime}(11)$.

\subsection{The generating functions for $C^{\prime \prime}(n)$ and $C_{1}^{\prime \prime}(n)$ :}

[Collected from Ramanujan's lost notebook and Andrews (1988)]

\subsubsection{The generating function for $C^{\prime \prime}(n)$ :}

$C^{\prime \prime}(n)$ is the number of partitions of $n$ into parts of the forms $5 m+2$ and $5 m+3$ given

In Table-1.11

$n \quad$ Partitions of $n$ into parts of the forms $5 m+2$ and $5 m+3 \quad C^{\prime \prime}(n)$

1 none 
It is convenient to define $C^{\prime \prime}(0)=1$.

We can write an expression for $C^{\prime \prime}(n)$ as;

$$
\begin{aligned}
& C^{\prime \prime}(0)+C^{\prime \prime}(1) x+C^{\prime \prime}(2) x^{2}+C^{\prime \prime}(3) x^{3}+C^{\prime \prime}(4) x^{4}+C^{\prime \prime}(5) x^{5}+\ldots \\
& =1+0 . x+x^{2}+x^{3}+x^{4}+x^{5}+2 x^{6}+2 x^{7}+\ldots \infty \\
& =\frac{1}{\left(1-x^{2}\right)\left(1-x^{3}\right)\left(1-x^{7}\right)\left(1-x^{8}\right) \ldots \infty} \quad \text { [Birman (1988)] } \\
& =\sum_{m=0}^{\infty} \frac{1}{\left(1-x^{5 m+2}\right)\left(1-x^{5 m+3}\right)} \\
& \therefore \sum_{m=0}^{\infty} \frac{1}{\left(1-x^{5 m+2}\right)\left(1-x^{5 m+3}\right)}=\sum_{n=0}^{\infty} C^{\prime \prime}(n) x^{n} .
\end{aligned}
$$

\subsubsection{The generating function for $C_{1}^{\prime \prime}(n)$ :}

$C_{1}^{\prime \prime}(n)$ is the number of partitions of $n$ into parts not less than 2 and with minimal difference 2 given in Table-1.12

$n \quad$ Partitions of $n$ into parts not less than 2 and with minimal $\quad C_{1}^{\prime \prime}(n)$ difference 2

$1 \quad$ none

We can write an expression for $C_{1}^{\prime \prime}(n)$ as;

$$
\begin{aligned}
& C_{1}^{\prime \prime}(0)+C_{1}^{\prime \prime}(1) x+C_{1}^{\prime \prime}(2) x^{2}+C_{1}^{\prime \prime}(3) x^{3}+C_{1}^{\prime \prime}(4) x^{4}+C_{1}^{\prime \prime}(5) x^{5}+\ldots \\
& =1+x^{2}+x^{3}+x^{4}+x^{5}+2 x^{6}+2 x^{7}+3 x^{8}+\ldots \infty
\end{aligned}
$$




$$
\begin{aligned}
& =1+\frac{x^{2}}{1-x}+\frac{x^{6}}{(1-x)\left(1-x^{2}\right)}+\frac{x^{12}}{(1-x)\left(1-x^{2}\right)\left(1-x^{3}\right)}+\ldots \infty \\
& =1+\sum_{m=1}^{\infty} \frac{x^{m(m+1)}}{(1-x)\left(1-x^{2}\right) \ldots\left(1-x^{m}\right)} \\
& \therefore 1+\sum_{m=1}^{\infty} \frac{x^{m(m+1)}}{(1-x)\left(1-x^{2}\right) \ldots\left(1-x^{m}\right)}=1+\sum_{n=1}^{\infty} C_{1}^{\prime \prime}(n) x^{n} .
\end{aligned}
$$

[Rankin (1989) and Lovejoy (2003)]

Theorem 1.3: The number of partitions of $n$ into parts not less than 2 and with minimal difference 2 is equal to the number of partitions of $n$ into parts of the forms $5 m+2$ and $5 m+3$

$$
\text { i.e., } C_{1}^{\prime \prime}(n)=C^{\prime \prime}(n) \text {. }
$$

Proof: From Ramanujan's Lost Notebook, we get;

$H_{k}(a, x)=\frac{G_{k}(a, x)}{(1-a)(1-a x) \ldots \infty},\left[\right.$ where $H_{0}=0$ and $k=1$ or 2$]$

If $k=1$ and $a=x$, we get;

$$
\begin{aligned}
H_{1}(x, x)=\frac{G_{1}(x, x)}{(1-x)\left(1-x^{2}\right)\left(1-x^{3}\right) \ldots \infty} & =\frac{\prod_{m=0}^{\infty}\left(1-x^{5 m+1}\right)\left(1-x^{5 m+4}\right)\left(1-x^{5 m+5}\right)}{(1-x)\left(1-x^{2}\right)\left(1-x^{3}\right) \ldots \infty}[\mathrm{by}(1.6 .3 \mathrm{c})] \\
& =\frac{1}{\left(1-\mathrm{x}^{2}\right)\left(1-x^{3}\right)\left(1-x^{7}\right)\left(1-x^{8}\right)\left(1-x^{12}\right)\left(1-x^{13}\right) \ldots} \\
& =\prod_{m=0}^{\infty} \frac{1}{\left(1-x^{5 m+2}\right)\left(1-x^{5 m+3}\right)} \\
o r, H_{1}(x, x) & =\prod_{m=0}^{\infty} \frac{1}{\left(1-x^{5 m+2}\right)\left(1-x^{5 m+3}\right)} \quad \ldots \quad \text { (1.6.3j) }
\end{aligned}
$$

Again, from Ramanujan's Lost Notebook, we get

$H_{k}-H_{k-1}=a^{k-1} \eta H_{3-k}$, where the operator $\eta$ is defined by $\eta f(a)=f(a x)$, and $k=1$ or 2 , then

$H_{1}=\eta H_{2}, H_{2}-H_{1}=a \eta H_{1}$.

So we have,

$H_{2}=\eta H_{2}+a \eta^{2} H_{2}$. $\quad \ldots$

We suppose that; $H_{2}=1+c_{1} a+c_{2} a^{2}+\ldots$

where the coefficients $c_{i}$ depend on $x$ only. Substituting this into (1.6.3k), we obtain;

$1+c_{1} a+c_{2} a^{2}+\ldots \infty=1+c_{1} a x+c_{2} a^{2} x^{2}+\ldots \infty+a\left(1+c_{1} a x^{2}+c_{2} a^{2} x^{4}+\ldots \infty\right)$.

Hence, equating the coefficients of various powers of a from both sides we get;

$c_{1}=\frac{1}{1-x}, c_{2}=\frac{x^{2}}{1-x^{2}} c_{1}, c_{3}=\frac{x^{4}}{1-x^{3}} c_{2}, \ldots, c_{n}=\frac{x^{n(n-1)}}{(1-x)\left(1-x^{2}\right) . .\left(1-x^{n}\right)}$.

Putting these values in(1.6.31) 
$H_{2}=H_{2}(a, x)=1+\frac{a}{1-x}+\frac{a^{2} x^{2}}{(1-x)\left(1-x^{2}\right)}+\frac{a^{3} x^{6}}{(1-x)\left(1-x^{2}\right)\left(1-x^{3}\right)}+\ldots \infty \ldots(1.6 .3 \mathrm{~m})$

From above we get; $H_{1}=\eta H_{2}$

$H_{1}=H_{1}(a, x)=\eta\left\{1+\frac{a}{(1-x)}+\frac{a^{2} x^{2}}{(1-x)\left(1-x^{2}\right)}+\frac{a^{3} x^{6}}{(1-x)\left(1-x^{2}\right)\left(1-x^{3}\right)}+\ldots\right\} \quad[\operatorname{by}(1.6 .3 \mathrm{~m})]$

$H_{1}=H_{1}(a, x)=\left\{1+\frac{a x}{(1-x)}+\frac{a^{2} x^{4}}{(1-x)\left(1-x^{2}\right)}+\frac{a^{3} x^{9}}{(1-x)\left(1-x^{2}\right)\left(1-x^{3}\right)}+\ldots\right\}$

If $a=x$, then; $H_{1}(x, x)=\left\{1+\frac{x^{2}}{(1-x)}+\frac{x^{6}}{(1-x)\left(1-x^{2}\right)}+\frac{x^{12}}{(1-x)\left(1-x^{2}\right)\left(1-x^{3}\right)}+\ldots\right\}$

From (1.6.3j) and (1.6.3n) we get;

$$
\begin{aligned}
& 1+\frac{x^{2}}{(1-x)}+\frac{x^{6}}{(1-x)\left(1-x^{2}\right)}+\frac{x^{12}}{(1-x)\left(1-x^{2}\right)\left(1-x^{3}\right)}+\ldots=\prod_{m=0}^{\infty} \frac{1}{\left(1-x^{5 m+2}\right)\left(1-x^{5 m+3}\right)} \\
& \text { i.e, } 1+\sum_{m=1}^{\infty} \frac{x^{m(m+1)}}{(1-x)\left(1-x^{2}\right) . .\left(1-x^{m}\right)}=\prod_{m=0}^{\infty} \frac{1}{\left(1-x^{5 m+2}\right)\left(1-x^{5 m+3}\right)}
\end{aligned}
$$

[It is also known as Rogers- Ramanujan's Identity]

$$
\therefore 1+\sum_{n=1}^{\infty} C_{1}^{\prime \prime}(n) x^{n}=1+\sum_{n=1}^{\infty} C^{\prime \prime}(n) x^{n} \text {. }
$$

Equating the co-efficient of $\mathrm{x}^{\mathrm{n}}$ from both side we get;

$$
C_{1}^{\prime \prime}(n)=C^{\prime \prime}(n)
$$

i.e., the number of partitions of $n$ into parts not less than 2 and with minimal difference 2 is equal to the number of partitions of $n$ into parts of the forms $5 m+2$ and $5 m+3$. Hence the Theorem.

\section{Example 1.4:}

If $n=11$, the four partitions of 11 into parts not less than 2 and with minimal difference 2 are given below:

$11,9+2,8+3,7+4$. Hence, $C_{1}^{\prime \prime}(11)=4$.

Again the four partitions of 11 into parts of the form $5 m+2$ and $5 m+3$ are given as;

$8+3,7+2+2,3+3+3+2,3+2+2+2+2$. Hence, $C^{\prime \prime}(11)=4$.

$$
\therefore C_{1}^{\prime \prime}(11)=C^{\prime \prime}(11) \text {. }
$$

\subsection{We discuss the generating functions for $P^{\prime}(n)$ and $P^{\prime \prime}(n)$ :}

[Collected from Ramanujan's lost notebook and Berndt (1991)]

\subsubsection{The generating function for $P^{\prime}(n)$ :}

$P^{\prime}(n)$ is the number of partitions of $n$ into parts of the form $n=a_{1}+a_{2}+\ldots+a_{r}$, where $a_{i}-a_{i+1} \geq 3$ and if $3 \mid a_{i}$, then $a_{i}-a_{i+1}>3$ given in Table-1.13

$$
\begin{array}{lll}
n & \text { Type of partitions } & P^{\prime}(n) \\
1 & 1 & 1
\end{array}
$$




$\begin{array}{lll}2 & 2 & 1 \\ 3 & 3 & 1 \\ 4 & 4 & 1 \\ 5 & 5,4+1 & 2 \\ \ldots & \ldots & \ldots\end{array}$

It is convenient to define $P^{\prime}(0)=1$.

We can write an expression for $P^{\prime}(n)$ as;

$$
\begin{aligned}
& P^{\prime}(0)+P^{\prime}(1) x+P^{\prime}(2) x^{2}+P^{\prime}(3) x^{3}+P^{\prime}(4) x^{4}+\ldots \\
& =1+x+x^{2}+x^{3}+x^{4}+2 x^{5}+2 x^{6}+3 x^{7}+3 x^{8}+\ldots \infty \\
& =(1+x)\left(1+x^{2}\right)\left(1+x^{4}\right)\left(1+x^{5}\right)\left(1+x^{7}\right) \ldots \quad \text { [Andrews (1979)] }=\prod_{n=0}^{\infty}\left(1+x^{3 n+1}\right)\left(1+x^{3 n+2}\right) . \\
& \therefore \prod_{n=0}^{\infty}\left(1+x^{3 n+1}\right)\left(1+x^{3 n+2}\right)=\sum_{n=0}^{\infty} P^{\prime}(n) x^{n} .
\end{aligned}
$$

\subsubsection{The generating function for $P^{\prime \prime}(n)$ :}

$P^{\prime \prime}(n)$ is the number of partitions of $n$ into parts congruent to $\pm 1(\bmod 6)$

given in Table- 1.14

$$
\begin{array}{lll}
n & \text { Type of partitions } & P^{\prime \prime}(n) \\
1 & 1 & 1 \\
2 & 1+1 & 1 \\
3 & 1+1+1 & 1 \\
4 & 1+1+1+1 & 1 \\
5 & 5,1+1+1+1+1 & 2
\end{array}
$$

It is convenient to define $P^{\prime \prime}(0)=1$,

We can write an expression for $P^{\prime \prime}(n)$ as; $\quad P^{\prime \prime}(0)+P^{\prime \prime}(1) x+P^{\prime \prime}(2) x^{2}+P^{\prime \prime}(3) x^{3}+P^{\prime \prime}(4) x^{4}+\ldots$

$$
\begin{aligned}
=1 & +x+x^{2}+x^{3}+x^{4}+2 x^{5}+2 x^{6}+3 x^{7}+3 x^{8}+. . \infty \\
= & \left(1+x+x^{2} . .\right)\left(1+x^{5}+x^{10} . .\right)\left(1+x^{7}+x^{14}+. .\right) . . \text { [Andrews (1979)] } \\
= & (1-x)^{-1}\left(1-x^{5}\right)^{-1}\left(1-x^{7}\right)^{-1} \ldots \\
= & \frac{1}{(1-x)\left(1-x^{5}\right)\left(1-x^{7}\right) . .} \\
= & \prod_{n=0}^{\infty} \frac{1}{\left(1-x^{6 n+1}\right)\left(1-x^{6 n+5}\right)} \\
& \quad \therefore \prod_{n=0}^{\infty} \frac{1}{\left(1-x^{6 n+1}\right)\left(1-x^{6 n+5}\right)}=\sum_{n=0}^{\infty} P^{\prime \prime}(n) x^{n} .
\end{aligned}
$$

Corollary 1.5: $P^{\prime}(n)=P^{\prime \prime}(n)$ 
Proof: From above we get;

$$
\begin{aligned}
& \sum_{n=0}^{\infty} P^{\prime}(n) x^{n}=\prod_{n=0}^{\infty}\left(1+x^{3 n+1}\right)\left(1+x^{3 n+2}\right) \\
= & (1+x)\left(1+x^{2}\right)\left(1+x^{4}\right)\left(1+x^{5}\right)\left(1+x^{7}\right)\left(1+x^{8}\right) \ldots \\
= & \frac{\left(1-x^{2}\right)\left(1-x^{4}\right)\left(1-x^{8}\right) \ldots}{(1-x)\left(1-x^{2}\right)\left(1-x^{4}\right) \ldots} \\
= & \frac{1}{(1-x)\left(1-x^{5}\right)\left(1-x^{7}\right)\left(1-x^{11}\right) \ldots} \\
= & \prod_{n=0}^{\infty} \frac{1}{\left(1-x^{6 n+1}\right)\left(1-x^{6 n+5}\right)}=\sum_{n=0}^{\infty} P^{\prime \prime}(n) x^{n} . \\
\therefore & \sum_{n=0}^{\infty} P^{\prime}(n) x^{n}=\sum_{n=0}^{\infty} P^{\prime \prime}(n) x^{n} .
\end{aligned}
$$

Equating the coefficient of $x^{n}$ from both sides we get;

$$
P^{\prime}(n)=P^{\prime \prime}(n) \text {. Hence the Corollary. }
$$

Now we can consider a Partition Theorem;

Theorem 1.4: $P^{\prime}(n)=P^{\prime \prime}(n)$. i.e., the number of partitions of $n$ into parts of the form $n=a_{1}+a_{2}+\ldots+a_{r}$, where $a_{i}-a_{i+1} \geq 3$ and if $3 \mid a_{i}$, then $a_{i}-a_{i+1}>3$ is equal to the number of partitions of $n$ into parts congruent to $\pm 1(\bmod 6)$.

Proof: We establish an one-to-one correspondence between the partitions enumerated by $P^{\prime}(n)$ and those enumerated by $P^{\prime \prime}(n)$. Firstly we consider partition enumerated by $P^{\prime}(n)$, let $n=a_{1}+a_{2}+\ldots+a_{r}$, where all terms congruent to $\pm 1(\bmod 6)$ except $a_{i}$ or $a_{j}$, where $i \in[1, r]$ and $j \in[1, r]$. If $a_{i}$ is multiple by 3 , then $a_{i}$ can be expressed the terms congruent to $1(\bmod 6)$ and $a_{j}$ can be expressed the terms congruent to $\pm 1(\bmod 6)$, like;

$9+2=1+1+1+1+1+1+1+1+1+1+1$, and $8+3=5+1+1+1+1+1+1$.

Now we are arranging all the terms of the partition of $n$ can be expressed the terms congruent to $\pm 1(\bmod 6)$. Consequently all the terms of the partition of $n$ can be enumerated by $P^{\prime}(n)$ can be converted to the partitions of $n$ into parts congruent to $\pm 1(\bmod 6)$. So, our correspondence is oneto-one.

Conversely, we transfer the partitions of $n$ enumerated by $P^{\prime \prime}(n)$. Let $n=a_{1}+a_{2}+\ldots+a_{r}$, where all terms congruent to $\pm 1(\bmod 6)$, we sum the terms in the $1^{\text {st }}$ group of $n$, it would be $a_{i}$ (say) where $i \in[1, r]$ and sum the terms in the $2^{\text {nd }}$ group of $n$, it would be $a_{i+1}$ (say), $a_{i}-a_{i+1} \geq 3$ and if $3 \mid a_{i}$, then $a_{i}-a_{i+1}>3$, like: $7+1+1+1+1=7+4,5+5+1=10+1$ and $5+1+1+1+1+1+1=$ 
$8+3$, then all parts of the partitions of $n$ into parts of the form $n=a_{1}+a_{2}+\ldots+a_{r}$, where $a_{i}-a_{i+1} \geq 3$ and if $3 a_{i}$, then $a_{i}-a_{i+1}>3$. Consequently all the partitions of $n$ enumerated by $P^{\prime \prime}(n)$ can be converted to the partitions of n enumerated by $P^{\prime}(n)$. Totally our correspondence is onto i.e., the number of partitions of $n$ into parts of the form $n=a_{1}+a_{2}+\ldots+a_{r}$, where $a_{i}-a_{i+1} \geq 3$ and if $3 \mid a_{i}$, then $a_{i}-a_{i+1}>3$ is equal to the number of partitions of $n$ into parts congruent to $\pm 1(\bmod 6)$.

$$
\text { i.e., } P^{\prime}(n)=P^{\prime \prime}(n) \text {. Hence the Theorem. }
$$

Numerical example 1.5: when $n=11$. If $n=11$, the five partitions of 11 that are enumerated by $P^{\prime}(n)$ are: $11,10+1,9+2,8+3$, and $7+4$. The five partitions of 11 into parts congruent to $\pm 1(\bmod 6)$ are $11,7+1+1+1+1,5+5+1,5+1+1+1+1+1+1$, and $1+1+1+1+1+1+1+1+1+1+1$.

$$
\text { i.e., } P^{\prime}(11)=P^{\prime \prime}(11) \text {. }
$$

1.10. Consider the Generating Functions For $P_{1}^{2}(n), P_{1}^{r}(n), P_{1}^{2}(n)$ and $P_{1}^{2}(n)$, with $\mathbf{r} \geq \mathbf{2}$ :

\section{[Collected from Ramanujan's lost notebook]}

\subsubsection{The Generating Function for $P_{1}^{2}(n)$ :}

$P_{1}^{2}(n)$ is the number of partitions of $n$ into parts that are either even and not congruent to 6 $(\bmod 8)$ or odd and congruent to $3,7(\bmod 8)$ given in Table-1.15

$\begin{array}{lll}n & \begin{array}{l}\text { Partitions of } n \text { into parts that are either even and not } \\ \text { congruent to } 6(\bmod 8) \text { or odd and congruent to } 3,7 \\ (\bmod 8)\end{array} & P_{1}^{2}(n) \\ 1 & \begin{array}{l}\text { none } \\ 2\end{array} & 2 \\ 3 & 3 & 0 \\ 4 & 4,2+2 & 1 \\ 5 & 3+2 & 1 \\ 6 & 4+2,3+3,2+2+2 & 2 \\ \ldots & \ldots \ldots & 3\end{array}$

It is convenient to define $P_{1}^{2}(0)=1$.

We can write an expression for $P_{1}^{2}(n)$ as;

$$
\begin{aligned}
& P_{1}^{2}(0)+P_{1}^{2}(1) x+P_{1}^{2}(2) x^{2}+P_{1}^{2}(3) x^{3}+P_{1}^{2}(4) x^{4}+P_{1}^{2}(5) x^{5}+\ldots \\
& =1+0 \cdot x+1 \cdot x^{2}+1 \cdot x^{3}+2 \cdot x^{4}+1 \cdot x^{5}+3 \cdot x^{6}+\ldots
\end{aligned}
$$


$=\frac{\left(1-x^{6}\right)\left(1-x^{14}\right) \ldots}{\left(1-x^{2}\right)\left(1-x^{3}\right)\left(1-x^{4}\right)\left(1-x^{6}\right)\left(1-x^{7}\right)\left(1-x^{8}\right) \ldots}[$ Andrews, (1967)]

$=\sum_{j=1}^{\infty} \frac{\left(1-x^{8 j-2}\right)}{\left(1-x^{2 j}\right)\left(1-x^{4 j-1}\right)}$

$\therefore \sum_{j=1}^{\infty} \frac{\left(1-x^{8 j-2}\right)}{\left(1-x^{2 j}\right)\left(1-x^{4 j-1}\right)}=1+\sum_{n=1}^{\infty} P_{1}^{2}(n) x^{n}$.

In general, we can write

$$
\begin{aligned}
& \sum_{j=1}^{\infty} \frac{\left(1-x^{4 r j-2}\right)}{\left(1-x^{2 j}\right)\left(1-x^{2 r j-1}\right)} \\
& =\prod_{j=1}^{\infty}\left(1-x^{4 r j-2}\right)\left(1+x^{2 j}+x^{4 j}+\ldots \infty\right)\left(1+x^{2 r j-1}+x^{4 r j-2}+\ldots \infty\right) \\
& =1+\sum_{n=1}^{\infty} P_{1}^{r}(n) x^{n} \\
& \therefore \sum_{j=1}^{\infty} \frac{\left(1-x^{4 r j-2}\right)}{\left(1-x^{2 j}\right)\left(1-x^{2 r j-1}\right)}=1+\sum_{n=1}^{\infty} P_{1}^{r}(n) x^{n}, \text { [Ramanathan }(1981
\end{aligned}
$$

where the coefficient $P_{1}^{r}(n)$ is the number of partitions of $n$ into parts that are either even and not congruent to $4 r-2(\bmod 4 r)$ or odd and congruent to $2 r-1,4 r-1(\bmod 4 r)$.

\subsubsection{The Generating Function for $P_{2}^{2}(n)$ :}

$P_{2}^{2}(n)$ is the number of partitions of $n$ into parts that are either even or else congruent to 3 (mod4) with the further restriction that only even parts may be repeated given in

Table-1.16

$n \quad$ Partitions of $n$ into parts that are either even or else $P_{2}^{2}(n)$ congruent to $3(\bmod 4)$ with the further restriction that only even parts may be repeated

$1 \quad$ none

33

$4 \quad 4,2+2$

$5 \quad 3+2$

$6 \quad 6,4+2,2+2+2$

It is convenient to define $P_{2}^{2}(0)=1$. 
We can write an expression for $P_{2}^{2}(n)$ as;

$$
\begin{aligned}
& P_{2}^{2}(0)+P_{2}^{2}(1) x+P_{2}^{2}(2) x^{2}+P_{2}^{2}(3) x^{3}+P_{2}^{2}(4) x^{4}+P_{2}^{2}(5) x^{5}+\ldots \\
& =1+0 . x+1 \cdot x^{2}+1 \cdot x^{3}+2 \cdot x^{4}+1 \cdot x^{5}+3 \cdot x^{6}+\ldots \\
& =\frac{\left(1+x^{3}\right)\left(1+x^{7}\right)\left(1+x^{11}\right) \ldots}{\left(1-x^{2}\right)\left(1-x^{4}\right)\left(1-x^{6}\right)\left(1-x^{8}\right) \ldots} \text { [Andrews (1967)] } \\
& =\sum_{j=1}^{\infty} \frac{\left(1+x^{4 j-1}\right)}{\left(1-x^{2 j}\right)} \\
& \therefore \sum_{j=1}^{\infty} \frac{\left(1+x^{4 j-1}\right)}{\left(1-x^{2 j}\right)}=1+\sum_{n=1}^{\infty} P_{2}^{2}(n) x^{n} .
\end{aligned}
$$

In general, we can write

$$
\begin{aligned}
& \prod_{j=1}^{\infty} \frac{\left(1+x^{2 r j-1}\right)}{\left(1-x^{2 j}\right)} \\
= & \prod_{j=1}^{\infty}\left(1+x^{2 r j-1}\right)\left(1+x^{2 j}+x^{4 j}+\ldots \infty\right) \\
= & 1+\sum_{n=1}^{\infty} P_{2}^{r}(n) x^{n}, \\
\therefore & \prod_{j=1}^{\infty} \frac{\left(1+x^{2 r j-1}\right)}{\left(1-x^{2 j}\right)}=1+\sum_{n=1}^{\infty} P_{2}^{r}(n) x^{n},
\end{aligned}
$$

where the coefficient $P_{2}^{r}(n)$ is the number of partitions of $n$ into parts that are either even and not congruent to $4 r-2(\bmod 4 r)$ or odd and congruent to $2 r-1,4 r-1(\bmod 4 r)$.

Corollary 1.6: $P_{1}^{r}(n)=P_{2}^{r}(n)$

Proof: From above we get;

$$
\begin{aligned}
1+\sum_{n=1}^{\infty} P_{1}^{r}(n) x^{n} & =\sum_{j=1}^{\infty} \frac{\left(1-x^{4 r j-2}\right)}{\left(1-x^{2 j}\right)\left(1-x^{2 r j-1}\right)} \\
\text { or, } \sum_{n=0}^{\infty} P_{1}^{r}(n) x^{n} & =\sum_{j=1}^{\infty} \frac{\left(1-x^{2 r j-1}\right)\left(1+x^{2 r j-1}\right)}{\left(1-x^{2 j}\right)\left(1-x^{2 r j-1}\right)} \\
& =\sum_{j=1}^{\infty} \frac{\left(1+x^{2 r j-1}\right)}{\left(1-x^{2 j}\right)} \\
& =1+\sum_{n=1}^{\infty} P_{2}^{r}(n) x^{n}, \quad \text { [by above] } \\
& =\sum_{n=o}^{\infty} P_{2}^{r}(n) x^{n},
\end{aligned}
$$

Equating the co-efficient of $\mathrm{x}^{\mathrm{n}}$ from both sides we get; $P_{1}^{r}(n)=P_{2}^{r}(n)$. Hence the Corollary.

Here we give a Theorem, which is related to the terms $P_{2}^{r}(n)$ and $P_{3}^{r}(n)$. 
Theorem 1.5: The number of partitions of $n$ into parts that are either even or odd congruent to $2 r-1(\bmod 2 r)$ with the further restriction that only even parts may be repeated is equal to the number of partitions of $n$ of the form $n=b_{1}+b_{2}+\ldots+b_{s}$, where $b_{i} \geq b_{i+1}$, and for $b_{i}$ odd $b_{i}-b_{i+1} \geq 2 r-1$. Where $P_{3}^{r}(n)$ denote the number of partitions of $n$ of the form $n=b_{1}+b_{2}+\ldots+b_{s}$, where $b_{i} \geq b_{i+1}$, and for $b_{i}$ odd, $b_{i}-b_{i+1} \geq 2 r-1 \quad\left(1 \leq i \leq s\right.$, where $\left.b_{s+1}=0\right)$.

$$
\text { i.e., } P_{2}^{r}(n)=P_{3}^{r}(n) \text {. }
$$

Proof: Let $\pi_{1}$ be a partition of the type enumerated by $P_{3}^{r}(n)$. We represent $\pi_{1}$ graphically with each even part $2 m$ represented by two rows of m nodes and each odd part $2 m+1$ represented by two rows of $m+1$ nodes and $m$ nodes respectively.

Such as $9+6$ becomes;

Now we may consider the graph vertically with the condition that $r$ columns are always to be grouped as a single part, whenever the lowest node in the most right hand column of the group is not presented there. If $r=2$, form above graph we obtain in this manner;

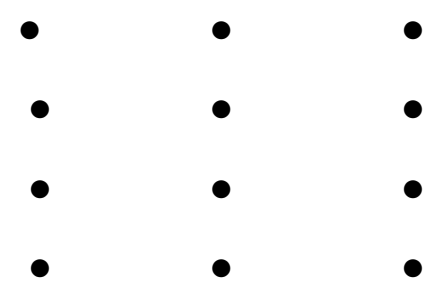

The partition $4+4+4+3$. Now since the condition on partitions enumerated by $P_{3}^{r}(n)$ is $b_{i}-b_{i+1} \geq 2 r-1$, whenever $b_{i}$ is odd. Thus a part congruent to $2 r-1(\bmod 2 r)$ is produced. Since originally odd parts were distinct, we see that now odd parts will be congruent to $2 r-1(\bmod 2 r)$ and will not be repeated and since originally all odd parts were greater or equal to $2 r-1$, we see that there will always be $r$ columns available for each grouping. Thus in this case we have produced a partition of the type enumerated by $P_{2}^{r}(n)$. Clearly our correspondence is one to one, however, the above process is reversible and thus the correspondence is onto. So that $P_{2}^{r}(n)=P_{3}^{r}(n)$.

Hence the Theorem.

Example 1.6: We take $r=2, n=9$. The corresponding partitions are listed opposite each other in the following table -1.17 :

$\begin{array}{lll}\begin{array}{l}\text { Type of partitions } \\ \text { enumerated by } P_{3}^{r}(9)\end{array} & \text { With relevant graph } & \begin{array}{l}\text { Type of partitions } \\ \text { enumerated by } P_{2}^{r}(9)\end{array} \\ 9 & \text { - } \bullet-~ & 2+2+2+3\end{array}$


$7+2$

$6+3$

$5+2+2$

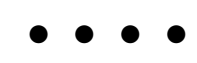

$+0$

$\bullet$

$\bullet \bullet$

$\bullet \bullet-$

$\bullet \bullet$

$\bullet \bullet \bullet$
$6+3$

$4+2+3$

$7+2$

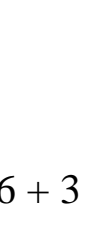

\section{$\bullet$}

\section{○}

Now we can write $P_{3}^{r}(9)=P_{2}^{r}(9)=4$.

1.11. The Generating Functions [Andrews et al. (2013)] for FFW (n) and $\sum_{n=1}^{\infty} F F W(z, n) x^{n}$ :

1.11.1 The generating Function for FFW (n):

FFW (n): Let D denote the set of partitions into distinct parts. We define;

$$
\operatorname{FFW}(\mathrm{n})=\sum_{\substack{\pi \in D \\|\pi|=n}}(-1)^{\#(\pi)-1} s(\pi),
$$

where $s(\pi)$ is the smallest part of a partition $\pi$, and \# $(\pi)$ is the number of parts given in Table-1.18

$\begin{array}{llll}n & \text { Partitions of n into distinct parts } & \mathrm{s}(\pi) & F F W(n) \\ 1 & 1 & 1 & 1 \\ 2 & 2 & 2 & 2 \\ 3 & 3,2+1 & 3,1 & 2 \\ 4 & 4,3+1 & 4,1 & 3\end{array}$



$5 \quad 5,4+1,3+2$
$5,1,2$
2
$6 \quad 6,5+1,4+2,3+2+1$
$6,1,2,1 \quad 4$

We can write an expression for $F F W(n)$ as;

$$
\begin{aligned}
& F F W(1) x+F F W(2) x^{2}+F F W(3) x^{3}+F F W(4) x^{4}+\ldots \\
& =x+2 x^{2}+2 x^{3}+3 x^{4}+2 x^{5}+4 x^{6}+ \\
& =\frac{x}{(1-x)(1-x)}+\frac{(-1) x^{3}}{(1-x)\left(1-x^{2}\right)\left(1-x^{2}\right)}+\frac{x^{6}}{(1-x)\left(1-x^{2}\right)\left(1-x^{3}\right)\left(1-x^{3}\right)^{+. .}} \quad \text { [Fokkink et al. (1995)] } \\
& =\sum_{n=1}^{\infty} \frac{(-1)^{n-1} x^{\frac{n+1}{2}}}{(x)_{n}\left(1-x^{n}\right)} \\
& \therefore \sum_{n=1}^{\infty} \frac{(-1)^{n-1} x^{\frac{n+1}{2}}}{(x)_{n}\left(1-x^{n}\right)}=\sum_{n=1}^{\infty} F F W(n) .
\end{aligned}
$$

Relation 2.1. $F F W(n)=\mathrm{d}(\mathrm{n})$

Proof: A relation related to the term $d(n)$.

We get; FFW (1) = $1=\mathrm{d}(1)$

FFW (2) $=2=\mathrm{d}(2)$

FFW (3) $=2=\mathrm{d}(3)$

FFW (4) $=3=\mathrm{d}(4)$

FFW $(5)=2=d(5)$

We can write the relation $F F W(n)=\mathrm{d}(\mathrm{n})$. Hence the Relation.

Corollary 2.7: $\frac{x}{(1-z x)(1-x)}=\sum_{k=1}^{\infty}\left(\frac{z^{k}-1}{z-1}\right) x^{k}$

Proof: L.H.S $=\frac{x}{(1-z x)(1-x)}$

$$
\begin{aligned}
& =x\left(1+z x+z^{2} x^{2}+z^{3} x^{3}+\ldots\right)\left(1+x+x^{2}+x^{3}+. .\right) \\
& =x+(1+z) x^{2}+\left(1+z+z^{2}\right) x^{3}+\left(1+z+z^{2}+z^{3}\right) x^{4}+\ldots \\
& =x+\frac{(1+z)(1-z)}{(1-z)} x^{2}+\frac{\left(1+z+z^{2}\right)(1-z)}{(1-z)} x^{3}+\frac{\left(1+z+z^{2}+z^{3}\right)(1-z)}{(1-z)} x^{4}+. . \\
& =x+\frac{\left(1-z^{2}\right)}{(1-z)} x^{2}+\frac{\left(1-z^{3}\right)}{(1-z)} x^{3}+\frac{\left(1-z^{4}\right)}{(1-z)} x^{4}+. \\
& =x+\frac{\left(z^{2}-1\right)}{(z-1)} x^{2}+\frac{\left(z^{3}-1\right)}{(z-1)} x^{3}+\frac{\left(z^{4}-1\right)}{(z-1)} x^{4}+.
\end{aligned}
$$


$=\sum_{k=1}^{\infty} \frac{\left(z^{k}-1\right)}{(z-1)} x^{k}=$ R.H.S. $\quad$ Hence the Corollary.

Corollary 2.8: $\sum_{n=1}^{\infty} F F W(z, n) x^{n}=\sum_{n=1}^{\infty} \frac{(-1)^{n-1} x^{\frac{n(n+1)}{2}}}{\left(1-z x^{n}\right)(x)_{n}}$

Proof: We get;

$\sum_{n=1}^{\infty} F F W(z, n) x^{n}=\sum_{n=1}^{\infty}\left(x^{n}+(1+z) x^{2 n}+\ldots . .+\left(1+z+\ldots+z^{k-1}\right) x^{k n}+\ldots\right) \frac{(-1)^{n-1} x^{\frac{n(n-1)}{2}}}{(x)_{n-1}}$

[Andrews et al (2013) and Andrews, Encycl. Math. (1985)]

$$
\begin{aligned}
& =\sum_{n=1}^{\infty}\left\{x^{n}+\frac{(1+z)(1-z)}{(1-z)} x^{2 n}+\frac{\left(1+z+z^{2}\right)(1-z) x^{3 n}}{(1-z)}+\ldots\right) \frac{(-1)^{n-1} x^{\frac{n(n-1)}{2}}}{(x)_{n-1}} \\
& =\sum_{n=1}^{\infty}\left\{x^{n}+\frac{z^{2}-1}{z-1} x^{2 n}+\frac{z^{3}-1}{z-1} x^{3 n}+\ldots .\right\} \frac{(-1)^{n-1} x^{\frac{n(n-1)}{2}}}{(x)_{n-1}} \\
& =\sum_{n=1}^{\infty}\left(\sum_{k=1}^{\infty} \frac{z^{k}-1}{z-1} x^{n k}\right) \frac{(-1)^{n-1} x^{\frac{n(n-1)}{2}}}{(x)_{n-1}}=\sum_{n=1}^{\infty} \frac{x^{n}}{\left(1-z x^{n}\right)\left(1-x^{n}\right)} \frac{(-1)^{n-1} x^{\frac{n(n-1)}{2}}}{(x)_{n-1}} \quad \text { [by above] } \\
& =\sum_{n=1}^{\infty} \frac{(-1)^{n-1} x^{\frac{n(n+1)}{2}}}{\left(1-z x^{n}\right)(x)_{n}} \\
& \text { [Since } \sum_{n=1}^{\infty}\left(1-x^{n}\right)(x)_{n-1}=(1-x)+\left(1-x^{2}\right)(1-x)+\left(1-x^{3}\right)\left(1-x^{2}\right)(1-x)+\ldots . .=\sum_{n=1}^{\infty}(x)_{n} \text { ] } \\
& \therefore \sum_{n=1}^{\infty} F F W(z, n) x^{n}=\sum_{n=1}^{\infty} \frac{(-1)^{n-1} x^{\frac{n(n+1)}{2}}}{\left(1-z x^{n}\right)(x)_{n}} \text {. Hence the Corollary. }
\end{aligned}
$$

Corollary 2.9: $\sum_{n=1}^{\infty} F F W(z, n) x^{n}=\sum_{k=0}^{\infty} \frac{z^{k}}{(x)_{k}}\left[(x)_{k}-(x)_{\infty}\right]$

Proof: We get;

$$
\begin{aligned}
& \sum_{\mathrm{n}=1}^{\infty} \operatorname{FFW}(\mathrm{z}, \mathrm{n}) x^{n}=\mathrm{x}+(1+\mathrm{z}) \mathrm{x}^{2}+\left(z+z^{2}\right) x^{3}+\left(z+z^{2}+z^{3}\right) x^{4}+\left(-1+z^{2}+z^{3}+z^{4}\right) x^{5}+\ldots . \\
& =x+x^{2}+z\left(x^{2}+x^{3}+x^{4}\right)+z^{2}\left(x^{3}+x^{4}\right)+z^{3} x^{4}+\ldots . \\
& =\left\{1-(x)_{\infty}\right\}+\frac{z}{(1-x)}\left\{(1-x)-(x)_{\infty}\right\}+\frac{z^{2}}{(1-x)\left(1-x^{2}\right)}\left\{(1-x)\left(1-x^{2}\right)-(x)_{\infty}\right\} \\
& +\frac{z^{3}}{(1-x)\left(1-x^{2}\right)\left(1-x^{3}\right)}\left\{(1-x)\left(1-x^{2}\right)\left(1-x^{3}\right)-(x)_{\infty}\right\}+\ldots . \\
& =\sum_{k=0}^{\infty} \frac{z^{k}}{(x)_{k}}\left[(x)_{k}-(x)_{\infty}\right] \text {. }
\end{aligned}
$$


$\therefore \sum_{n=1}^{\infty} F F W(z, n) x^{n}=\sum_{k=0}^{\infty} \frac{z^{k}}{(x)_{k}}\left[(x)_{k}-(x)_{\infty}\right]$. Hence the Corollary.

Corollary 2.10: FFW $(1, \mathrm{n})=\mathrm{FFW}(\mathrm{n})$

Proof: We get; $\quad \sum_{n=1}^{\infty} \frac{(-1)^{n-1} x^{\frac{n(n+1)}{2}}}{(x)_{n}\left(1-z x^{n}\right)}$

$=\mathrm{x}+(1+z) x^{2}+\left(z+z^{2}\right) x^{3}+\left(z+z^{2}+z^{3}\right) x^{4}+\left(-1+z^{2}+z^{3}+z^{4}\right) x^{5}+\left(-1+1+z^{2}+z^{3}+z^{4}+z^{5}\right) x^{6}+\ldots$.

Or, $\sum_{n=1}^{\infty} F F W(z, n) x^{n}=\mathrm{x}+(1+\mathrm{z}) \mathrm{x}^{2}+\left(z+z^{2}\right) x^{3}+\left(z+z^{2}+z^{3}\right) x^{4}+\left(-1+z^{2}+z^{3}+z^{4}\right) x^{5}+\ldots .$.

If $\mathrm{z}=1$, we get;

$\sum_{n=1}^{\infty} F F W(1, n) x^{n}=\mathrm{x}+2 \mathrm{x}^{2}+2 x^{3}+3 x^{4}+2 x^{5}+4 x^{6}+\ldots$. $=\sum_{n=1}^{\infty} \frac{(-1)^{n-1} x^{\frac{n(n+1)}{2}}}{(x)_{n}\left(1-x^{n}\right)} \quad[$ by above $]$

$\sum_{n=1}^{\infty} F F W(1, n) x^{n} \quad=\sum_{n=1}^{\infty} F F W(n) x^{n}$.

Equating the co-efficient of $x^{n}$ form both sides we get;

$\therefore$ FFW $(1, n)=$ FFW $(n)$.Hence the Corollary.

\section{CONCLUSION}

In this article we have shown $C_{1}^{\prime}(n)=C^{\prime}(n)$ with the help of a numerical example when $n=11$, and have shown $C_{1}^{\prime \prime}(n)=C^{\prime \prime}(n)$ with the help of a numerical example when $n=11$. We have proved the Theorem $P_{1}^{r}(n)=P_{3}^{r}(n)$. for any positive integer of $n$ and $r \geq 2$. In this article we have found the number of partitions of $\mathrm{n}$ into distinct parts with required conditions. We have proved the Corollaries containing a pair of generating functions

$\sum_{n=1}^{\infty} \frac{(-1)^{n-1} x^{\frac{n(n+1)}{2}}}{(x)_{n}\left(1-z x^{n}\right)}, \frac{1}{(1-z)}\left\{1-\frac{(x)_{\infty}}{(z x)_{\infty}}\right\}$ and $\sum_{k=0}^{\infty} \frac{z^{k}}{(x)_{k}}\left[(x)_{k}-(x)_{\infty}\right], \sum_{k=0}^{\infty} z^{k}\left\{1-\left(x^{k+1} ; x\right)_{\infty}\right\}$

by simplifications. We have established the Corollary FFW $(1, \mathrm{n})=\mathrm{FFW}(\mathrm{n})$ by taking $\mathrm{z}=1$.

\section{ACKNOWLEDGMENT}

It is great pleasure to express my sincerest gratitude to my respected professor Md. Fazlee Hossain, Department of Mathematics, University of Chittagong, Bangladesh. 


\section{REFERENCES}

[1] Andrews, G. E. "The Theory of Partitions, Encycl. Math. Appl." Vol.2, Addison-Wesley Reading Mass,(1976), (Reissued, Cambridge University press, Cambridge,1985).

[2] Andrews, G. E., Garvan, F.G. and J. Liang, " Self-Conjugate Vector Partitions and the Parity of the spt-function", Acta, Arith., 158, (2013),pp. 199-218.

[3] Andrews, G. E." On Schur's Second Partition Theorem”, Glasgow Math. J.8, (1967), pp. 127-132.

[4] Andrews, G. E. “An Introduction to Ramanujan's Lost Notebook”, Amer. Math. Monthly, 86,(1979), pp. 89-108.

[5] Andrews, G. E. "Ramanujan's Lost Notebook VIII: the entire Rogers-Ramanujan function” Advances in Mathematics, 191, (2005), pp. 393-407.

[6] Andrews, G. E. “An Introduction to Ramanujan's Lost Notebook and other unpublished papers”, Norosa Publishing House, New Delhi, (1988), pp.1-120.

[7] Birman,J. S. (Columbia University), “Bulletin (New series) of the American Mathematical Society, Vol.19, No.2, Oct. (1988), pp.558-559.

[8] Berndt, B.C. “Ramanujan's Notebook, Part III”, Springer-Verlag, New York, (1991). p. 69.

[9] Das, S. “Generating Functions For “, American Rev. of Mathematics and Statistics, Vol.1, No.1, Dec.(2013), pp.38-40.

[10] Das,S. “Some Restricted Partition Functions”, American J. of Applied Mathematics and Statistics, Vol.2, No.6, (2014), pp. 386-393.

[11] Fokkink, R., Fokkink, W. and Wang, Z.B. "A relation between partitions and the number of divisors",Amer. Math. Monthly, 102, (1995),pp. 345-346.

[12] Hardy, G. H. and Ramanujan, S." Asymptotic formulae in combinatory analysis", February 28 ${ }^{\text {th }}$, (1917), pp.75-79.

[13] Lovejoy,J. “Note on Gordon's Theorem for Overpartitions”, J. of Combinatorial Theory, Series A, 103, (2003), pp.393-401

[14] Rankin, R. A. “Ramanujan's manuscripts and Notebooks, II”, Bull. London Math. Soc. 21, (1989), pp.351-365.

[15] Ramanathan, K. G. “The Unpublished Manuscripts of Srinivasa Ramanujan”, Tata Institute of Fundamental Research, Bombay, India, Vol. 50, No.5, March 5,(1981),pp.187-197.

[16] Ramanujan, S. “ The lost notebook and other unpublished papers”, Spriger-Verlag, (1988), pp.1-76.

[17] Ramanujan, S. “The lost notebook and other unpublished papers", (Intro. By G. E. Andrews), Norosa, New Delhi, (1988), p.76.

[18] Toh,P. C. "Ramanujan type identities and congruences for partition pairs", Unimodular. Net/ Archivel Pairs. pdf, Oct.11, (2011), pp.01-09. 